\title{
Reducing the impact of inhomogeneous broadening on quantum dot based electromagnetically induced transparency
}

\author{
Hansen, Per Lunnemann; Mørk, Jesper
}

\section{Published in:}

Applied Physics Letters

Link to article, DOI:

10.1063/1.3079676

Publication date:

2009

Document Version

Publisher's PDF, also known as Version of record

Link back to DTU Orbit

Citation (APA):

Hansen, P. L., \& Mørk, J. (2009). Reducing the impact of inhomogeneous broadening on quantum dot based electromagnetically induced transparency. Applied Physics Letters, 94(7), 071108.

https://doi.org/10.1063/1.3079676

\section{General rights}

Copyright and moral rights for the publications made accessible in the public portal are retained by the authors and/or other copyright owners and it is a condition of accessing publications that users recognise and abide by the legal requirements associated with these rights.

- Users may download and print one copy of any publication from the public portal for the purpose of private study or research.

- You may not further distribute the material or use it for any profit-making activity or commercial gain

- You may freely distribute the URL identifying the publication in the public portal

If you believe that this document breaches copyright please contact us providing details, and we will remove access to the work immediately and investigate your claim 


\title{
Reducing the impact of inhomogeneous broadening on quantum dot based electromagnetically induced transparency
}

\author{
Per Lunnemann ${ }^{\text {a) }}$ and Jesper Mørk ${ }^{\text {b) }}$ \\ DTU Fotonik, Technical University of Denmark, DK-2800 Kgs Lyngby, Denmark
}

(Received 1 December 2008; accepted 17 January 2009; published online 18 February 2009)

\begin{abstract}
Slow light based on electromagnetically induced transparency in an inhomogeneously broadened quantum dot medium is investigated theoretically. Three schemes, $\Xi, \mathrm{V}$, and $\Lambda$, are compared and it is shown that the V-scheme gives a group velocity that is more than three orders of magnitude smaller compared to the $\Xi$ - and $\Lambda$-schemes. The physical mechanisms that make the V-scheme less vulnerable to inhomogeneous broadening are analyzed and discussed. () 2009 American Institute of Physics. [DOI: 10.1063/1.3079676]
\end{abstract}

Electromagnetically induced transparency (EIT) was in 1999 proven effective at drastically reducing the speed of light in an ultracold atomic gas. ${ }^{1}$ The result has since then given rise to a number of impressive experiments based on EIT. $^{2}$ Quantum dots (QDs) are excellent candidates for EIT in semiconductor material due to their quantized energy levels and relatively low dephasing rates, ${ }^{3}$ as well as the possibility for monolithic integration. In order to obtain a significant slowdown, it is necessary to interact with an ensemble of QDs. However, self-assembled QDs exhibit inhomogeneous broadening (IHB), mainly caused by size fluctuations of the QD. At cryogenic temperatures, IHB is several orders of magnitude wider than the transition linewidth. ${ }^{4}$ The broadening causes the probe and coupling laser fields to experience different detunings depending on the individual QD size and, as a result, the overall EIT features are smeared out. EIT was previously demonstrated in quantum well structures by exploiting excitonic effects ${ }^{5}$ and, in Ref. 6, a similar scheme was used in QDs by selectively addressing a subpopulation of the QD ensemble. In this work we suggest a simple scheme that effectively reduces the detrimental effect of IHB while allowing for coupling and probe beams of similar wavelengths.

Three types of generic schemes, $\Xi, \mathrm{V}$, and $\Lambda$, are commonly used for EIT (see Fig. 1). We note that for a spherical potential, the transition $|1\rangle \rightarrow|3\rangle$, shown in Fig. 1, is a forbidden transition as a result of orthogonality of the envelope functions. However, band mixing and strain effects relax these selection rules, thus allowing a finite coupling between some of the otherwise forbidden transitions, although with a somewhat weaker coupling. Another way of increasing the coupling strength could be to apply a bias voltage across the QD layer in order to break the symmetry of the envelope functions.

EIT is a coherent effect that renders an otherwise absorbing medium transparent to the probe when applying a strong coupling field. ${ }^{2}$ The effect can be interpreted as arising from destructive interference between two absorption paths. For the $\Lambda$-scheme, this corresponds to the two paths, $|1\rangle \rightarrow|3\rangle$ and $|1\rangle \rightarrow|3\rangle \rightarrow|2\rangle \rightarrow|3\rangle$, that we shall refer to as single- and two-photon pathways, respectively. The rapid change in the absorption near resonance of the probe correspondingly

\footnotetext{
a) Electronic mail: plha@fotonik.dtu.dk.

${ }^{b)}$ Electronic mail: jesm@fotonik.dtu.dk.
}

causes a change in the group index and we shall in the following investigate the possible reduction in the group velocity of the three schemes in an inhomogeneously broadened QD medium.

Calculations of the slowdown factor, defined as the group index relative to the background index $n_{\mathrm{g}} / n_{\mathrm{bg}}$, were based on a density matrix approach similar to Ref. 7 . For the calculations we use $1 / \Gamma_{21}=1 / \Gamma_{31}=500 \mathrm{ps}, 1 / \Gamma_{32}=8 \mathrm{~ns}$, and $1 / \gamma_{21}=1 / \gamma_{31}=1 / \gamma_{32}=600 \mathrm{ps}$, where $\Gamma_{i j}$ and $\gamma_{i j}$ denote the population decay and dephasing rate, respectively, between state $|i\rangle$ and $|j\rangle$. To date, no measurement has been published, giving the measured population decay and dephasing rates on the same QD sample for transitions related to those depicted in Fig. 1. Thus, the used population decay rates are similar to those measured in Ref. 4, while the dephasing rate $\gamma_{21}$ is similar to Ref. 8 at $T=7 \mathrm{~K}$. We tentatively set $\gamma_{31}$ and $\gamma_{32}$ equal to $\gamma_{21}$. The chosen relaxation time, $1 / \Gamma_{32}$, is long compared to typical values reported $(\sim 1-10 \mathrm{ps})$. As will be discussed, a low intraband relaxation time is required to achieve EIT for the V-scheme. However, QDs with such long intraband relaxation time can in fact be fabricated, as demonstrated in Ref. 4. For comparison, we also calculate the slowdown factor of the $\mathrm{V}$-scheme with a short intraband relaxation time $1 / \Gamma_{32}=10 \mathrm{ps}$ and with a population decay limited dephasing time. For the dipole moments $\mu_{i j}$, we use $\mu_{13} / e=7 \AA, \mu_{12} / e=1 \AA$, and $\mu_{23} / e=47 \AA$, where $e$ is the electron charge. For ease of notation, we shall denote the dephasing and population decay related to the optically coupled transitions as $\gamma_{p}$ and $\Gamma_{p}$ for the probe and $\gamma_{c}$ and $\Gamma_{c}$ for the coupling transition, respectively. For the transition between terminal states that are not coupled, dephasing and population decay is denoted as $\gamma_{t}$ and $\Gamma_{t}$, respectively. A similar notation is used for the dipole moments, i.e., $\mu_{p}$, $\mu_{c}$, and $\mu_{t}$. The laser detunings are measured relative to the peak of the inhomogeneous distribution and we use a confinement factor $\Gamma=6 \times 10^{-3}$. Finally, the inhomogeneous dis-
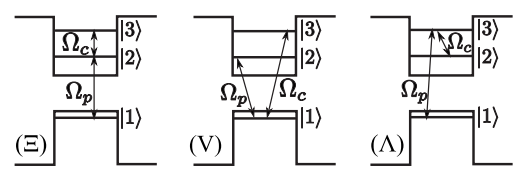

FIG. 1. Illustration of the three different excitation schemes considered in the QD. $\Omega_{p}$ and $\Omega_{c}$ denote the Rabi frequency of the probe and coupling laser field, respectively. 


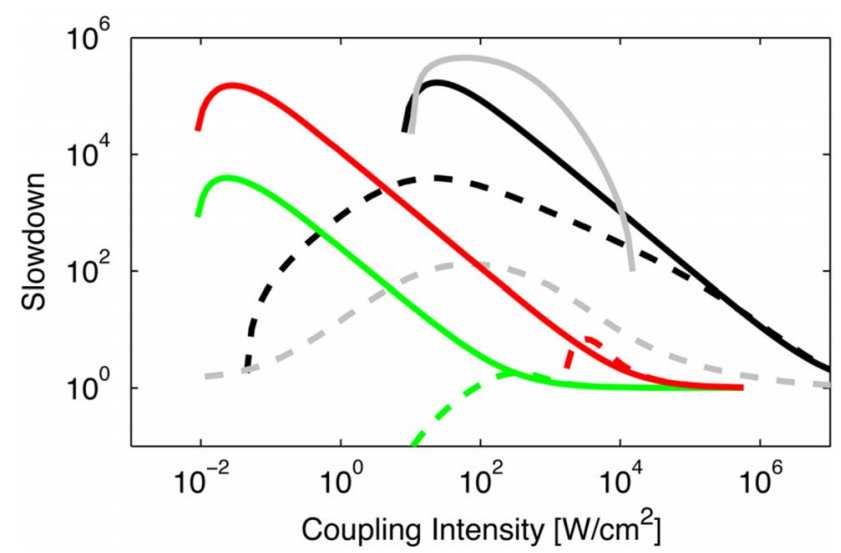

FIG. 2. (Color online) Calculations of the slowdown factor for the three schemes V (black and gray), $\Lambda$ (green), and $\Xi$ (red). Solid/dashed lines are with/without IHB taken into account. All plots are for long intraband relaxation time, $\Gamma_{12}^{-1}=8 \mathrm{~ns}$, except for the gray plots where $\Gamma_{12}^{-1}=10 \mathrm{ps}$.

tribution function is assumed to be Gaussian and for the purpose of illustration we choose a full width at half maximum of $\sigma_{\text {ih }}=200 \gamma_{p} \sim 0.44 \mathrm{meV}$, where we recognize this value to be somewhat smaller than typical QD samples $(\sim 10 \mathrm{meV})$.

The slowdown factor is shown in Fig. 2 as a function of the coupling laser intensity for all three schemes. Looking at the results without IHB taken into account (dashed lines), it is seen that the $\mathrm{V}$-schemes require a higher coupling intensity due to the much lower dipole moment of the coupling transition. However, when looking at the results including IHB (solid lines), it is seen that the V-scheme is vastly superior, with a maximum slowdown of $\sim 4 \times 10^{3}$ at $20 \mathrm{~W} / \mathrm{cm}^{2}$. For the other schemes, signs of slowdown are almost completely washed out. Increasing the IHB to realistic values $(\sim 10 \mathrm{meV})$, it was observed that the maximum slowdown factor decreases to $\approx 200$ for the V-scheme, while for the other schemes all slowdown is lost. To understand what causes this notable difference, we need to analyze the mechanisms behind EIT a little closer.

It can be shown ${ }^{2}$ that transparency occurs when the probe and coupling detunings fulfill the two-photon resonance condition $\delta \equiv \Delta_{p} \pm \Delta_{c}=0$, where the sum is for the $\Xi$-scheme and the difference is for the V-and $\Lambda$-schemes and $\Delta_{p}\left(\Delta_{c}\right)$ is the probe (coupling) laser detuning with respect to the probe (coupling) transition. In case the pump is detuned far away from resonance, only the probe pathway contributes and as a result, normal absorption occurs. We shall denote this as the primary absorption resonance. ${ }^{9}$ Consider instead both lasers far detuned, but on two-photon resonance. As mentioned, here the absorption terms from the single- and two-photon pathways exactly cancel. If the probe frequency is now slightly further detuned, transition amplitudes from the single- and two-photon paths no longer cancel, and as a result absorption occurs with the dominant contribution arising from the two-photon pathway. We denote this as the secondary absorption resonance.

The absorption and refractive index of the probe for a given class of QDs are found from the imaginary and real part of the electric susceptibility $\chi_{p}\left(\widetilde{\Delta}_{p}, \widetilde{\Delta}_{c}\right)$ that in turn is calculated from the density matrix equations. ${ }^{7}$ Here we explicitly write the effective detuning of $\chi_{p}$, where $\widetilde{\Delta}_{p} \equiv \Delta_{p}$ $-\Delta_{p, i h}$, with $\Delta_{p}$ being the probe detuning and $\Delta_{p, i h}$ the fre-

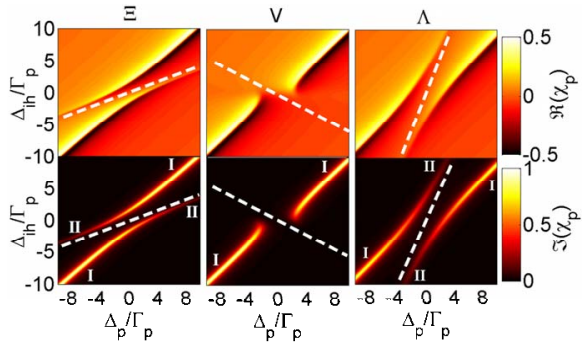

FIG. 3. (Color online) Real (top row) and imaginary (bottom row) parts of the electric susceptibility [in units of $\left.2 \Gamma \mu_{p}^{2} /\left(V \epsilon_{0} \hbar\right)\right]$ for the $\Xi$ - (left), V(middle), and $\Lambda$-schemes (right). Dashed white lines indicate the twophoton resonance $(\delta=0)$. Primary and secondary absorption peaks are indicated by I and II, respectively.

quency shift of the probe transition for a particular class of QDs. Both terms are measured relative to the center frequency of the inhomogeneous distribution of the probe transition. Similarly, $\widetilde{\Delta}_{c} \equiv \Delta_{c}-\Delta_{c, i h}$ for the coupling transition. The total material response is found by integrating $\chi_{p}\left(\widetilde{\Delta}_{p}, \widetilde{\Delta}_{c}\right)$ over $\Delta_{p, i h}$ and $\Delta_{c, i h}$ weighted by the inhomogeneous distribution functions of the two transitions. Modeling the QDs as disk shaped infinite potentials with radius $a$ and height $\ell$, we can explicitly derive the relation between $\Delta_{c, i h}$ and $\Delta_{p, i h}{ }^{3}$ Considering only fluctuations of $a$, a small change $\delta a$ in size implies a shift of the two lowest eigenenergies given by $\delta \varepsilon_{l}=-\hbar^{2} Z_{1 l}^{2} /\left(a^{3} m_{e}^{*}\right) \delta a$, where we assumed that $\ell<a$ (Ref. 3) and $l=0,1$. Here, $m_{e}^{*}$ is the electron effective mass and $Z_{1 l}$ denotes the first root of the $l$ th Bessel function. Due to the larger hole mass we shall in the following neglect shifts in the valence band. The change in eigenenergies causes a shift of both the probe $\left(\delta E_{p}\right)$ and the coupling $\left(\delta E_{p}\right)$ transition energies that depend on the chosen scheme. Defining $\kappa \equiv \delta E_{c} / \delta E_{p}$, the effective detuning of the pump and probe for a single QD is reduced to $\widetilde{\Delta}_{p}$ $=\Delta_{p}-\Delta_{i h}$ and $\widetilde{\Delta}_{c}=\Delta_{c}-\kappa \Delta_{i h}$, respectively, where $\Delta_{i h} \equiv \Delta_{p, i h}$. A plot of the normalized electric susceptibility as a function of $\Delta_{p}$ and $\Delta_{i h}$ is seen in Fig. 3 with $\Omega_{p}=5 \Gamma_{p}, \Delta_{c}=0,1 / \Gamma_{21}$ $=1 / \Gamma_{31}=500 \mathrm{ps}, 1 / \Gamma_{32}=8 \mathrm{~ns}$, and pure dephasing is neglected (dephasing is population decay limited).

The two-photon resonance condition for a QD class spectrally shifted by $\Delta_{i h}$ can then be put in the form $\Delta_{i h}$ $=(1 / 1 \pm \kappa) \Delta_{p} \equiv \alpha \Delta_{p}$ with the sum being for the $\Xi$-scheme and the difference for the $\mathrm{V}$ - and $\Lambda$-schemes. This relation is indicated on Fig. 3 as dashed white lines.

Looking at the absorption (bottom row) it is seen that close to the two-photon resonance the secondary absorption resonance peak appears, while the primary absorption resonance peak is located along the line $\Delta_{i h}=\Delta_{p}$, as expected. One notable difference is that the slope $\alpha$ of the two-photon resonance is negative for the $\mathrm{V}$-scheme, while it is positive for the other two schemes. For $\Xi$ and $\Lambda$, this causes the secondary resonance to cross the region where $\Delta_{p}=0$. Thus, when calculating the average absorption, EIT is destroyed. This, however, is not the case for the V-scheme where the negative value of $\alpha$ ensures that the secondary resonance peak never crosses the transparency region. This is also reflected in the calculated slowdown factor in Fig. 2 where, for the $\Xi$ - and $\Lambda$-schemes, the coupling power needs to be large enough for the Rabi splitting to overcome the width of the IHB. The nature of quantum confinement ensures $\alpha \leq 0$ for 
the considered V-scheme, while $\alpha \geq 0$ for the $\Xi$ - and $\Lambda$-scheme; we therefore stress that this observed difference remains valid in the case of using a more realistic model of the QD. Furthermore, it is seen from the figure that the $\mathrm{V}$-scheme lacks clear signatures of the secondary absorption resonance. This stems from the fact that in the V-scheme the coupling transition involves carrier excitations as opposed to the other two schemes. ${ }^{9}$ For those QDs where the coupling laser is far off resonance (i.e., $\left|\Delta_{i h}\right| \gg 0$ ) carriers from $|1\rangle$ are no longer excited to $|3\rangle$ and as a result absorption from the two-photon pathway vanishes. Of course, this also implies a reduction in the number of QDs contributing to the slowdown. Comparing the slowdown factor of the V-scheme in Fig. 2 for a 10 ps intraband relaxation time (gray) to that with an 8 ns relaxation time (black), it is seen that an effective slowdown remains, although with somewhat smaller magnitude. However, for the short relaxation time, coherence between the terminal states $|2\rangle$ and $|3\rangle$ is lost and the calculated slowdown is thus no longer caused by EIT. Instead, the probe transition exhibits gain that is being pumped by the pump field. The spectral width of the gain profile leads to a slowdown factor larger than unity. Increasing all of the interlevel dephasing rates inevitably decreases the effect of EIT on all schemes. However, the V-scheme was consistently observed to show the largest slowdown as long as the IHB is much broader than the dephasing rates. It is interesting to note that when $\Omega_{c} \leq \Gamma_{c}$ in the $\mathrm{V}$-scheme, a reduction in the absorption is still present. However, the effect no longer stems from EIT but is rather due to spectral hole burning by the coupling field. This is revealed in Fig. 2 for intensities below $10 \mathrm{~W} / \mathrm{cm}^{2}$, where the slowdown for the $\mathrm{V}$-scheme with IHB is seen to drop off much slower for decreasing coupling intensity compared to the case without IHB.

The reduced impact of IHB for the V-scheme opens the possibility of exploiting EIT in a waveguide structure, where the probe propagates along the QD growth plane. This could be implemented by using a simple ridge waveguide for confinement, thereby drastically increasing the interaction length. Furthermore, the coupling laser is of similar wavelength, which opens the possibility for simultaneously using the waveguide for the coupling laser. However, one must take into account that the coupling laser in this case is being absorbed along the waveguide. Thus, an optimal waveguide length exists beyond which the probe starts to experience severe absorption, while no further delay is achieved.

In conclusion, we theoretically investigated the three schemes, $\Xi, \Lambda$, and $\mathrm{V}$, for realizing EIT in an inhomogeneously broadened QD medium. IHB was shown to be detrimental for the $\Xi$ - and $\Lambda$-scheme, whereas for the V-scheme this was effectively eluded. This arises from two effects. First, the V-scheme has a two-photon resonance condition with a negative slope between the probe detuning and inhomogeneous spectral shift $\left(\Delta_{i h}\right)$. Second, the V-scheme relies on excitation of carriers, which thereby diminishes the influence from QDs off resonance.

This work was supported by the Danish Research Councils in the framework of QUEST as well as by the EU through the IST project QPHOTON.

${ }^{1}$ L. V. Hau, S. E. Harris, Z. Dutton, and C. H. Behroozi, Nature (London) 397, 594 (1999).

${ }^{2}$ M. Fleischhauer, A. Imamoglu, and J. P. Marangos, Rev. Mod. Phys. 77, 633 (2005).

${ }^{3}$ J. Kim, S. L. Chuang, P. C. Ku, and C. J. Chang-Hasnain, J. Phys.: Condens. Matter 16, S3727 (2004).

${ }^{4}$ R. Heitz, H. Born, F. Guffarth, O. Stier, A. Schliwa, A. Hoffmann, and D. Bimberg, Phys. Rev. B 64, 241305 (2001).

${ }^{5}$ M. C. Phillips, H. Wang, I. Rumyantsev, N. H. Kwong, R. Takayama, and R. Binder, Phys. Rev. Lett. 91, 183602 (2003).

${ }^{6}$ S. Marcinkevičius, A. Gushterov, and J. P. Reithmaier, Appl. Phys. Lett. 92, 041113 (2008).

${ }^{7}$ P. K. Nielsen, H. Thyrrestrup, J. Mørk, and B. Tromborg, Opt. Express 15, 6396 (2007).

${ }^{8}$ P. Borri, W. Langbein, S. Schneider, U. Woggon, R. L. Sellin, D. Ouyang, and D. Bimberg, Phys. Rev. Lett. 87, 157401 (2001).

${ }^{9}$ J. R. Boon, E. Zekou, D. McGloin, and M. H. Dunn, Phys. Rev. A 59, 4675 (1999) 